\title{
MITOS EN LA INVESTIGACIÓN CUALITATIVA
}

\section{MYTHS IN QUALITATIVE RESEARCH}

\author{
John Wesley Taylor V \\ Asociación General de los Adventistas del Séptimo Día, EE.UU. \\ taylorjw@gc.adventist.org \\ https://orcid.org/0000-0001-5673-6808
}

\begin{abstract}
RESUMEN
Se examinan cuatro mitos que se encuentran a menudo dentro de la investigación cualitativa y que tienen el potencial de distorsionar los resultados del esfuerzo y de la efectividad de los investigadores. Estos mitos deben ser deconstruidos y reemplazados por sus realidades.
\end{abstract}

Palabras clave: mitos, investigación cualitativa

\section{ABSTRACT}

Four myths that are often found in qualitative research and have the potential to distort the results of the effort and effectiveness of researchers are examined. These myths must be deconstructed and replaced by their realities.

Keywords: myths, qualitative research

\section{Introducción}

Entre sus varios sentidos, un mito puede definirse como una creencia ampliamente difundida, pero falsa. Fábulas, fantasías, falsas noticias e ilusiones abundan en toda cultura y en la sociedad contemporánea. Desde tiempos antiguos, sin embargo, las Escrituras señalan el peligro de aceptar mitos sin fundamento. Escribiendo a sus protegidos, Timoteo y Tito, el apóstol Pablo advirtió que no deberían abandonar la verdad, corriendo tras ideas engañosas ( 2 Timoteo 4:4), sino mostrar solidez al dejar de prestar atención a mitos y fábulas (Tito 1:14).

¿Podría ser que haya mitos relacionados con la investigación cualitativa? Desde la perspectiva del investigador cualitativo, ciertamente existen mitos con respecto a la misma investigación cualitativa, tales como los siguientes: la investigación cualitativa no es científica, la investigación cualitativa es totalmente subjetiva, la investigación cualitativa es meramente descriptiva y lo cualitativo simplemente no es confiable (Athanasiou, Debas y Darzi, 2010; Denzin y Lincoln, 2018; Green y Thorogood, 2014; Osborne, 2008; Stake, 2010; Waller, Farquharson y Dempsey, 2016).

Si bien se han proporcionado respuestas que creemos confrontan y resuelven efectivamente esos mitos externos, como investigadores cualitativos también debemos preguntarnos: ¿podría haber también mitos dentro de la misma investigación cualitativa, 
falsas creencias que distorsionan nuestra visión de la realidad y que nos llevan a formar conceptos erróneos sobre el enfoque cualitativo y sus metodologías? En este artículo, consideramos cuatro de esos mitos internos.

\section{Mito \# 1: El investigador debe abordar un tema cualitativo con una tabula rasa}

$\mathrm{He}$ aquí la premisa fundamental: debo olvidar lo que sé para descubrir lo que necesito saber. El resultado de aceptar esa suposición es la creencia de que un investigador cualitativo debe convertirse en un tablero en blanco, sin ideas preconcebidas $\mathrm{u}$ opiniones personales (Flick, 2014; Given, 2008; Urquhart, 2013). En consecuencia, el investigador debe lanzarse a la recolección de datos como recipiente no contaminado. $\mathrm{O}$ sea, sin antes revisar la literatura.

El origen de esa premisa se encuentra en un principio válido de la perspectiva naturalista, a saber, que un investigador cualitativo debe dejar de lado la teoría existente para evitar conclusiones prematuras (Hays y Singh, 2012; Lichtman, 2013; Trauth, 2001). Sin embargo, dejar de lado temporalmente la teoría no implica que un investigador cualitativo deba ignorar la literatura ni las construcciones teóricas, convirtiéndose en una tabula rasa. De hecho, jno puedes dejar de lado lo que no tienes!

El problema parece radicarse en una lectura superficial de la literatura sobre la investigación cualitativa. Desde un principio, Glaser y Strauss (1967) advirtieron a los investigadores que no permitiesen que teorías ya existentes dictasen lo que sería relevante en un estudio cualitativo. Sin embargo, tomar esa advertencia como un dictamen que requiere una mente en blanco es una interpretación errónea (Urquhart y Fernández, 2013).

El concepto de "dejar de lado la teoría existente" implica que el investigador comprende tanto el papel del conocimiento como del desapego. En ese escenario, las teorías conocidas o propuestas simplemente se dejan en reserva para una posible comparación futura, en caso de que el análisis de los datos indique que puedan ser relevantes. Eso permite al investigador acceder al conocimiento existente para aclarar el problema y diseñar el estudio, sin quedar atrapado en una opinión de lo que deberá representar la respuesta final.

Ahora al hecho. Un investigador cualitativo debe emplear, entonces, una revisión bifacética de la literatura (Urquhart y Fernández, 2013). La primera fase es un repaso sin compromiso de la literatura. En esta revisión preliminar, el investigador escanea la literatura para desarrollar tanto una sensibilidad teórica, como también comprender la naturaleza y forma de la investigación, buscando definir más claramente el problema e informándose sobre metodologías apropiadas.

En consecuencia, hay dos subcomponentes en esta revisión preliminar: (a) el investigador cualitativo se esfuerza por informarse sobre teorías existentes y señala aquellas que parecen ser potencialmente relevantes para futuras comparaciones, y (b) el investigador intenta descubrir cómo otros investigadores han abordado aspectos del problema o de situaciones similares, no para limitarse a ellas, sino para aprender de los logros y de los errores de otros.

La segunda fase, un repaso integrador de la literatura, toma lugar después de que la recopilación de datos ha comenzado. Durante esta fase se llevan a 
cabo dos formas de revisión, temática y teórica.

En la revisión temática de la literatura, que ocurre una vez que el análisis de datos está en marcha y los temas comienzan a surgir, el investigador vuelve a la literatura existente para desarrollar los conceptos emergentes. El enfoque es buscar literatura convergente y divergente para compararla con patrones observados y temas emergentes.

En esencia, la literatura se trata como fuente adicional de datos temáticos para enriquecer los conceptos emergentes del estudio. Además, al comparar patrones o conceptos nacientes con la literatura, el investigador puede llegar a reconocer la necesidad de muestreo adicional. En consecuencia, la revisión temática tiene que desempeñar un papel clave en robustecer la calidad de los conceptos emergentes.

En el repaso teórico de la literatura, que tiene lugar una vez que se han identificado los temas y se ha definido un patrón medular (es decir, una teoría sustantiva), el investigador busca integrarlos con las teorías relevantes ya existentes, sea para apoyarlas, para modificarlas o para agregar nuevas dimensiones. En esencia, esta revisión vincula los fenómenos observados con las teorías ya declaradas en campos similares, e incluso, potencialmente con metateorías en campos distantes.

En resumen, la primera fase de la revisión de la literatura es una revisión sin compromiso, donde el investigador busca (a) desarrollar sensibilidad teórica y (b) comprender la naturaleza y la forma de la investigación. Esto es seguido por una revisión integradora de la literatura, donde el investigador se esfuerza por (a) enriquecer la comprensión temática y (b) vincular la teoría sustantiva a la existente. Los elementos de un repaso cualitativo de la literatura pueden verse entonces como un proceso, ilustrado en la Figura 1.

Existe, sin embargo, una tensión inherente entre la forma en la cual los investigadores cualitativos trabajan con la literatura y el formato en que tradicionalmente se presentan las investigaciones en revistas académicas (Urquhart y Fernández, 2013).

\section{Teoría existente}

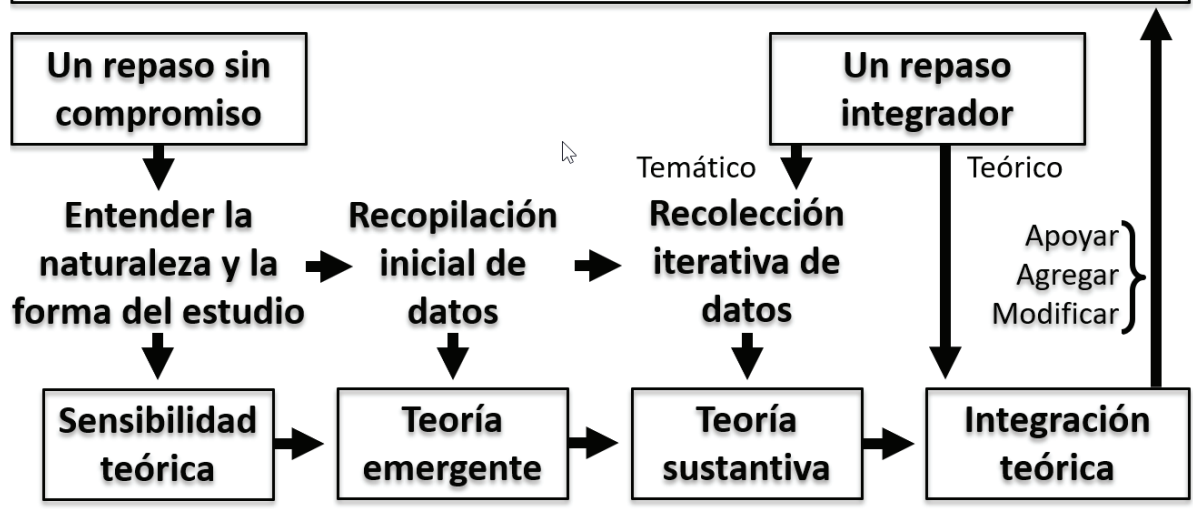

Figura 1. Relación de la teoría existente con el proceso de investigación cualitativa. 
Si la literatura se discute al principio del informe, los autores cualitativos pueden sentir que eso no representa la manera en que se incorporó la literatura a través del estudio. Pero si la literatura se presenta más adelante en el informe, es posible que el lector no tenga la información necesaria para comprender y evaluar adecuadamente el estudio. Además, el informe en sí puede correr el riesgo de ser rechazado por ciertas fuentes de publicación.

Una solución a este dilema puede ser presentar al principio del informe los resultados del repaso sin compromiso que sirvió para guiar la naturaleza y forma de la investigación y que proporcionó sensibilidad teórica. Luego, en la sección de discusión del estudio, se puede presentar literatura de la revisión integradora que enriqueció la comprensión temática y que vinculó los hallazgos del estudio con la teoría existente (Suddaby, 2006).

Al fin de todo, la conclusión es esta: realizar una investigación cualitativa no es excusa para ignorar la literatura.

\section{Mito \# 2: Los resultados de la investigación cualitativa no son generalizables}

El problema radica en que los investigadores cuantitativos a veces (probablemente, ja menudo!) descartan los resultados de la investigación cualitativa con base en la falta percibida de generalización. Los investigadores cualitativos generalmente responden que, de todos modos, no les preocupa la generalización, solamente la recopilación inmersiva de datos, la descripción detallada y la bondad de ajuste. ¿El resultado? ¡Pocos investigadores cuantitativos se convencen! (Gheondea-Eladi, 2014).

La validez - la veracidad de los ha- llazgos - y la confiabilidad — su grado de consistencia - son los estándares definitivos en investigación. Cada uno de estos criterios tiene dimensiones internas y externas. Por ejemplo, la confiabilidad (o la fidedignidad, como a menudo se le hace referencia en el paradigma cualitativo) tiene una dimensión interna de congruencia entre evaluadores y una dimensión externa de replicabilidad. La validez interna, por otro lado, se centra en la coincidencia entre las construcciones del marco conceptual y la instrumentación (en una forma muy similar a la que establece el concepto cualitativo de credibilidad), mientras que la validez externa se refiere a la generalización, al grado al cual los resultados de la investigación son aplicables a otras configuraciones.

La generalización, sin embargo, puede definirse de varias maneras: como "un acto de razonamiento que implica extraer conclusiones amplias de casos particulares" (Polit y Beck, 2010), como "una inferencia sobre lo no observado basado en lo observado" (GheondeaEladi, 2014), y como una "afirmación de lo que es el caso en un lugar o tiempo, será así por igual en otro lugar o tiempo" (Payne y Williams, 2005). Otros señalan que la generalización no solamente se preocupa por extender conclusiones de una muestra a una población, sino también de un caso a otro caso (Bryman, 2008). En general, la generalización implica que los resultados de un estudio serán aplicables a través del tiempo, espacio y caso.

Fundamentalmente, hay tres formas en que se puede establecer la generalización: generalización estadística, generalización analítica y transferibilidad (Firestone, 1993; Onwuegbuzie y Leech, 2009; Polit y Beck, 2010). La generalización 
estadística se basa en una muestra que es representativa de su marco de muestreo, un hecho que permite al investigador generalizar los resultados a ese marco más amplio. Sin embargo, surge un problema cuando el investigador, en realidad, desea aplicar los hallazgos a la población total, de los cuales el marco de muestreo es solamente una parte, y del cual puede o no ser representativo.

Para ilustrar, supongamos que un investigador desea estudiar las diferencias de capacidad espacial entre adolescentes que son diestra-dominantes y los que son zurdos. El investigador extrae una muestra aleatoria de adolescentes que son estudiantes de secundarias públicas en un área metropolitana determinada. Los hallazgos derivados de esa muestra son aplicables, por extensión, a los estudiantes de las secundarias públicas en esa área metropolitana. Sin embargo, concluir que esos hallazgos son aplicables a la población total de todo adolescente sería una generalización excesiva y no fundamentada. Los adolescentes en las escuelas privadas, o en las escuelas primarias o preparatorias, o en las zonas rurales u otras regiones pueden ser similares, o también pueden ser muy diferentes. Simplemente no lo sabemos.

En consecuencia, se han dado advertencias en cuanto a la generalización estadística (Shadish, Cook y Campbell, 2002). En primer lugar, una cantidad considerable de estudios cuantitativos han utilizado una muestra de conveniencia u otra estrategia de muestreo no representativa. En segundo lugar, incluso cuando se utiliza una muestra verdaderamente aleatoria (o bien estratificada), el investigador generalmente se esfuerza por aplicar los hallazgos a la población total, en lugar de limitar la aplicación de los hallazgos al marco de muestreo.
Cualquiera de esas situaciones anula la generalización estadística.

En la transferibilidad, el investigador proporciona una descripción amplia y detallada, que se incluye en el informe de investigación recibido por el profesional lector, que luego puede hacer aplicación de los hallazgos al contexto local en función de las similitudes que percibe entre la descripción de la investigación y las características del entorno actual. Esta transferencia de hallazgos es, por supuesto, un tipo de generalización, en la cual los hallazgos se transfieren a través del tiempo, espacio y caso. Dicha generalización se ha descrito en ocasión como una generalización moderatum (Payne y Williams, 2005), dado que la responsabilidad recae sobre el investigador de proporcionar tanta información contextual como sea posible para ayudar al lector, como profesional, a identificar las características relevantes que pueden transferirse a otro contexto.

También hay precauciones, por supuesto, con respecto a la transferibilidad. Primero, el investigador, quien comprende mejor que nadie los hallazgos del estudio, no tiene un papel directo en su aplicación. En segundo lugar, generalmente no hay un ciclo de retroalimentación o verificación cruzada de si el lector, que a veces puede conocer poco sobre la metodología cualitativa, ha aplicado adecuadamente los resultados del estudio a las circunstancias del contexto local, estableciendo así la bondad de ajuste.

La generalización analítica se refiere a una generalización hacia la teoría, en lugar de una generalización a una población o a un entorno específico (Halkier, 2011; Neergaard y Leitch, 2015). Este tipo de generalización se fundamenta en la forma en como las construcciones que 
emergen de los datos se relacionan entre sí y cómo se relacionan con la teoría existente. En el último caso, hay varias posibilidades: (a) los resultados pueden apoyar una teoría existente, (b) los hallazgos pueden agregar nuevas dimensiones a la teoría existente, o (c) los resultados pueden modificar significativamente la teoría existente.

Para resumir, la generalización estadística aplica los hallazgos a una población o, más apropiadamente, al marco muestral. Esa forma de generalización se usa principalmente en la investigación cuantitativa. La transferibilidad (generalización moderada) aplica los hallazgos de un entorno específico a otro contexto similar y se utiliza principalmente en investigaciones cualitativas. La generalización analítica, sin embargo, aplica los hallazgos hacia una teoría y puede usarse tanto en la investigación cuantitativa como cualitativa.

Entonces, ¿cómo hacemos para deconstruir el mito de que la investigación cualitativa no es generalizable, incluso para aquellos que pueden argumentar, aunque de manera incorrecta, que la transferibilidad no es una forma de generalización? En pocas palabras, al utilizar la generalización analítica en la investigación cualitativa (Gheondea-Eladi, 2014; Maxwell y Chmiel, 2014).

Con todo esto, fenómenos algo sorprendentes tienen lugar en la investigación cualitativa. Primero, los investigadores cualitativos rara vez discuten la generalización de sus hallazgos, y segundo, si acaso lo comentan, rara vez argumentan que la generalización analítica es un propósito medular de su esfuerzo. Sin embargo, si no hay generalización, estamos llevando a cabo meramente una investigación-acción, en la que los hallazgos se limitan al entorno de recopilación de datos, y en la que el investigador y el profesional suelen ser uno y el mismo. Si bien la investigación-acción puede utilizar metodologías cualitativas, así como cuantitativas, una investigación-acción no es lo mismo que una investigación académica cualitativa (Meyer, 2000).

¿Cómo, entonces, podemos incorporar la generalización analítica? De dos maneras, principalmente: (a) a través de nuestra estrategia de muestreo y (b) a través de nuestro enfoque en el análisis de los datos (Gheondea-Eladi, 2014).

En términos de la estrategia de muestreo, necesitamos utilizar un muestreo intencional, en lugar de un muestreo casual de conveniencia o de bola de nieve. Estas últimas estrategias deberían reservarse para pruebas piloto y quizás para poblaciones de difícil acceso, como los usuarios de drogas ilegales. El muestreo intencional, por lo contrario, se orienta por consideraciones teóricas y eso permite hacer inferencias de las instancias particulares a una teoría más general.

En términos del enfoque del análisis de datos, la investigación cualitativa puede usar codificación ascendente o descendente. En la codificación ascendente, iniciamos con los datos mismos y empleamos una abstracción de los esquemas de codificación (es decir, los temas emergentes) para desarrollar una teoría que surge de los datos, que luego se somete a prueba con otros datos muestreados. Este enfoque se utiliza principalmente en la investigación cualitativa de teoría fundamentada. En la codificación descendente, partimos desde la teoría y usamos esquemas formales de codificación para evaluar esa teoría dentro de los datos. Normalmente, este enfoque se debería emplear en las otras formas de investigación cualitativa. Ambos, sin embargo, 
vinculan la teoría con los hallazgos del estudio.

En resumen, dado que la investigación cualitativa puede y debe ser generalizable, una discusión abierta sobre la generalización debería ser normativa en la presentación de informes de investigación cualitativa. Esta discusión debe abordar los métodos de muestreo y codificación empleados y su relación con la validez externa. El informe debe abordar específicamente la generalización analítica, así como el potencial de transferibilidad.

\section{Mito \# 3: Los grupos focales, si es que se utilizarán en la investigación cualitativa, deberán seguir criterios formalmente establecidos}

Hay al menos un par de falsas creencias que comprenden este mito. La primera creencia sostiene que, para ser efectivos, los grupos focales deben seguir "el plan". Este plan establece lo siguiente:

1. Los grupos focales deben configurarse a seis o siete participantes, para que todos puedan recibir el tiempo adecuado para expresarse.

2. Los grupos focales deben ser demográficamente homogéneos, para que los encuestados se sientan cómodos y hablen libremente.

3. Los moderadores deben decir poco y seguir el guion, de modo que los resultados puedan compararse entre diversos grupos focales (Kitzinger, 1995; Langer, 1999).

La segunda falsa creencia sostiene que en la era de la minería en las redes sociales, los grupos focales son simplemente obsoletos (Heist, 2013; Killian Branding, 2017; Pfanner, 2006; Schelmetic, 2016). En la era primitiva, cuando las personas realmente hablaban en- tre sí, los grupos focales constituían una de las pocas herramientas en el repertorio naciente del investigador cualitativo. Ahora, en la nueva era de la tecnología móvil y el big data, hemos llegado tanto más allá que los grupos focales no son más que una nota al pie de la historia de la investigación cualitativa.

¡Disparates! He aquí los hechos, con cinco conceptos básicos que se unen para presentar el caso (Breen, 2007; Krueger, 2002; Langer, 1999; Millward, 2012). En primer lugar, los grupos focales continúan siendo una forma clave de triangulación en la investigación cualitativa. Hay al menos dos razones para ese rol. La primera es que los grupos focales brindan acceso a grupos clave de encuestados. Si bien las redes sociales y los dispositivos móviles constituyen desarrollos importantes en la recopilación de datos cualitativos, las entrevistas y los grupos focales pueden incorporar la visión de segmentos que de otro modo podrían ignorarse. La segunda razón es que los grupos focales permiten una recopilación de datos más receptiva y extensa. Esto se debe a la naturaleza interactiva e iterativa de los grupos focales, que permite la ramificación y el sondeo.

En segundo lugar, el número ideal de encuestados en un grupo focal depende de muchos factores, tales como el perfil de encuestado, el tema y el estilo del moderador. Si bien es generalmente cierto que cuanto más pequeño el grupo, menor la sinergia y la energía en el grupo, los mini grupos focales de cuatro o cinco personas también pueden ser apropiados para ciertos tipos de encuestados, tales como víctimas del acoso, que poco participarían en grupos de mayor número.

Tercero, los grupos focales efectivos pueden ser tanto heterogéneos como 
homogéneos. Un investigador cualitativo puede usar grupos homogéneos para (a) mejorar el nivel de comodidad de los encuestados en asuntos sensibles o íntimos, (b) proporcionar a los encuestados la libertad de hablar libremente sobre aquellos que no están en el grupo y (c) permitir al investigador la oportunidad de comparar varios tipos de encuestados de grupo a grupo. Por el otro lado, el investigador también puede optar por usar grupos heterogéneos para (a) estudiar cómo interactúan los diferentes tipos de encuestados entre sí, (b) evitar el "pensamiento grupal" donde los miembros simplemente aceptan una opinión dominante y (c) ahorrar tiempo y recursos comparado con lo requerido para formar grupos de cada tipo demográfico.

Cuarto, los moderadores de grupos focales, a veces, deberán tomar un papel de liderazgo. La idea de que un moderador deberá decir poco solamente es cierta en un mundo ideal donde los encuestados hablan uno a la vez, se atienen al tema, no monopolizan la conversación y brindan explicaciones breves y detalladas. ¡Esto rara vez sucede! En el mundo real, el rol de un moderador es activo: proporcionan enfoque y cadencia, gestionan la dinámica del grupo, ofrecen comentarios no-direccionales y buscan detalles adicionales. En esencia, los moderadores deben ser tanto creativos como receptivos para ser efectivos.

En quinto lugar, los moderadores efectivos usan guías para discusión, no guiones. Un guion es un protocolo de investigación que debe seguirse textualmente, como receta inmutable. Una guía de discusión es una herramienta de investigación que se implementa con flexibilidad. La guía de discusión general- mente incluye una lista general de temas, una secuencia sugerida y posibles ramificaciones y sondeos. En un grupo focal, un estilo de "aprovechar el momento" a menudo se siente más espontáneo y conversacional, y es más probable que los encuestados se abran. Sin embargo, cada grupo tiene su propio ritmo y el investigador debe tratar de aprovechar su energía. Si los encuestados, por ejemplo, se entusiasman con un tema y el moderador los interrumpe y pasa a otro asunto, la energía se disipa y el grupo no la podrá recuperar fácilmente.

Si en una investigación cualitativa de mercado, por ejemplo, se encuentra que una idea o producto lleva a un rechazo instantáneo por parte de los encuestados, el investigador probablemente deba explorar lo que subyace a esa reacción: ¿Existe ansiedad con respecto al cambio? ¿Hay necesidad de información adicional? ¿Hay rechazo de aspectos específicos? ¿Qué es lo que podría traer aceptación?

Como investigadores, debemos recordar que la potencia de la investigación cualitativa es su flexibilidad dinámica. Algunos aspectos resultarán ricos; otros menos. Pueden surgir nuevas ideas, que luego pueden incorporarse a la guía de discusión. Podemos descubrir que sea necesario reformular preguntas para aclarar áreas de confusión. Y, por supuesto, las reacciones de los encuestados pueden alterar el mismo enfoque.

¿El resultado final de todo esto? Los grupos focales han sido y continuarán siendo una estrategia flexible y valiosa para la recopilación de datos cualitativos. Es importante recordar que no abandonamos a los viejos amigos solo porque nos hemos ganado uno nuevo. 
Mito \# 4: La investigación cualitativa conlleva escasos riesgos inherentes, si acaso los hubiera, sea para el investigador o para los participantes

Nuevamente, hay al menos dos falsas creencias que sustentan este mito. Primero, debido a que un investigador cualitativo busca ser neutral e imparcial, la creencia es que la experiencia de la investigación en sí no le afectará. Segundo, debido a que el investigador se esfuerza por minimizar cualquier efecto del proceso sobre el entorno, la esperanza es que el riesgo para los participantes sea mínimo.

Para deconstruir este mito, deberíamos quizás contrastar los paradigmas cuantitativo y cualitativo, en términos de la relación del conocedor con lo conocido. En el paradigma positivista de la investigación cuantitativa, el investigador debe mantenerse alejado. Lo ideal es un estudio in vitro, con su contexto cuidadosamente controlado. En el paradigma naturalista de la investigación cualitativa, el investigador debe estar inmerso. Lo ideal es un estudio in vivo en el entorno natural.

Supongamos por un momento que nos hemos propuesto estudiar la personalidad de los leones. Sí, las bestias de presa. Un científico in vitro estudiará al león tras las rejas. El científico in vivo, no obstante, estudiará al león en la sabana, sin ninguna barrera entre ellos. Ciertamente, jno hay riesgo para el investigador en un estudio cualitativo!

El hecho es que no solo existen riesgos físicos para la investigación cualitativa, sino que también riesgos significativos para la salud mental (Bloor, Fincham y Sampson, 2007; Dickson-Swift, James, Kippen y Liamputtong, 2008). ¿Cómo se verá personalmente afectado el investigador, por ejemplo, al realizar entrevistas y participar en las observaciones? Lo que verá o escuchará podría resultar en "conocimiento de culpabilidad", tal como información sobre delitos involuntarios o planeados. Estos dilemas éticos son reales y no infrecuentes.

En consecuencia, cada investigador cualitativo debe tener una persona de confianza, alguien que sea el consejero del investigador sobre asuntos éticos o de trauma personal durante un estudio. Y esto debe determinarse de antemano. No todos los problemas pueden predecirse con precisión y saber a quién uno acudirá en caso de dificultades puede brindarle alivio y minimizar la angustia.

En términos de los participantes, todos los riesgos percibidos de antemano deben darse a conocer. Si bien los investigadores cualitativos no dañarían a sabiendas a sujetos humanos, aún puede haber riesgos importantes involucrados. Estos pueden incluir estrés psicológico, pérdida de estatus, responsabilidades legales, ostracismo o repercusiones políticas o económicas (Hadjistavropoulos y Smythe, 2010; Morse, 2001; Sanjari, Bahramnezhad, Fomani, Shoghi y Cheraghi, 2014). Es responsabilidad ética del investigador cualitativo realizar una evaluación de riesgos y luego revelar cualquier riesgo conocido en el consentimiento informado.

El investigador cualitativo también debe proteger cuidadosamente el derecho a la privacidad. Como investigadores, respetamos y protegemos la privacidad de los participantes al evitar invasiones sutiles. Por ejemplo, no debemos grabar entrevistas en dispositivos ocultos. También debemos proteger las identidades de los sujetos mediante el uso de seudónimos y desenfoque facial en cualquier imagen, e igualmente evitar 
el uso de cualquier posible identificador en los informes de investigación. Cualquier excepción a estos parámetros debe ser expresamente autorizada por los participantes por escrito.

En resumen, los riesgos para los participantes y para el investigador, que son reales en toda investigación cualitativa, deben ser reconocidos y minimizados.

\section{Conclusión}

En este artículo hemos examinado cuatro mitos que se encuentran a menudo dentro de la investigación cualitativa y que tienen el potencial de distorsionar los resultados de nuestro esfuerzo y nuestra efectividad como investigadores. Estos mitos deben ser deconstruidos y reemplazados por sus realidades.

Primer mito: el investigador debe abordar un tema cualitativo con una pizarra en blanco. El hecho: un investigador cualitativo debe utilizar una revisión bifacética de la literatura.

Segundo mito: los resultados de la investigación cualitativa no son generalizables. El hecho: los investigadores cualitativos deberían utilizar la generalización analítica, además de la transferibilidad.

Tercer mito: los grupos focales, si fuesen a utilizarse rara vez en la investigación cualitativa, deben seguir criterios bien establecidos. El hecho: los grupos focales continúan siendo una estrategia cualitativa eficaz y flexible.

Cuarto mito: la investigación cualitativa conlleva pocos riesgos inherentes, si acaso los hubiera, sea para el investigador o los participantes. El hecho: existen riesgos para los participantes y para el investigador que deben ser reconocidos y minimizados.

¿Que los mitos se desmoronen y los hechos perduren!

\section{Referencias}

Athanasiou, T., Debas, H. y Darzi, A. (Eds.). (2010). Key topics in surgical research and methodology. Berlín: Springer-Verlag.

Bloor, M., Fincham, B. y Sampson, H. (2007). QUALITI (NCRM) commissioned inquiry into the risk to well-being of researchers in qualitative research. Recuperado de https://www .cardiff.ac.uk/socsi/qualiti/CIReport.pdf

Breen, R. L. (2007). A practical guide to focus-group research. Journal of Geography in Higher Education, 30(3), 463-475. https://doi .org/10.1080/03098260600927575

Bryman, A. (2008). Social research methods (2 ed.). Nueva York: Oxford University Press.

Denzin, N. K. y Lincoln, Y. S. (Eds.). (2018). The $S A G E$ handbook of qualitative research (5a ed.). Thousand Oaks, CA: Sage.

Dickson-Swift, V., James, E. L., Kippen, S. y Liamputtong, P. (2008). Risk to researchers in qualitative research on sensitive topics: Issues and strategies. Qualitative Health Research, 18(1), 133-144. https://doi .org/10.1177/1049732307309007

Firestone, W. A. (1993). Alternative arguments for generalizing from data as applied to qualitative research. Educational Researcher, 22, 16-23. https://doi.org/10.3102/0013189X022004016

Flick, U. (2014). An introduction to qualitative research ( $5^{\mathrm{a}}$ ed.). Los Ángeles: Sage.

Gheondea-Eladi, A. (2014). Is qualitative research generalizable? Journal of Community Positive Practices, 14(3), 114-124.

Given, L. (Ed.). (2008). The SAGE encyclopedia of qualitative research methods (Vol. 2). Londres: Sage.

Glaser, B. G. y Strauss, A. L. (1967). The discovery of grounded theory: Strategies for qualitative research. Nueva York, NY: Aldine.

Green, J. y Thorogood, N. (2014). Qualitative methods for health research (3aㅡ. ed.). Londres: Sage.

Hadjistavropoulos, T. y Smythe, W. E. (2010). Elements of risk in qualitative research. Ethics \& Behavior, 11(2), 163-174. https://doi .org/10.1207/S15327019EB1102_4

Halkier, B. (2011). Methodological practicalities in analytical generalization. Qualitative Inquiry, 17(9), 787-797. https://doi.org/ 10.1177/1077800411423194

Hays, D. G. y Singh, A. A. (2012). Qualitative inquiry in clinical and educational settings. Nueva York: The Guilford Press.

Heist, G. (2013). Five things that will become obsolete in MR sooner than you think. Recuperado de http://www.greenbookblog.org/2013/ 07/09/5-things-that-will-become-obsolete-inmr-sooner-than-you-think/ 


\section{TAYLOR}

Killian Branding. (2017). Are focus groups obsolete? Recuperado de http://www.killianbranding .com/whitepaper/are-focus-groups-obsolete/

Kitzinger, J. (1995). Qualitative research: Introducing focus groups. The British Medical Journal, 311, 299-302. https://doi.org/10.1136/ bmj.311.7000.299

Krueger, R. A. (2002). Designing and conducting focus group interviews. Recuperado de http://www .eiu.edu/ihec/Krueger-FocusGroupInterviews .pdf

Langer, J. (1999). 15 myths of qualitative research: It's conventional, but is it wisdom? Marketing News, 33(5), 13-14.

Lichtman, M. (2013). Qualitative research in education: A user's guide. Thousand Oaks, CA: Sage.

Maxwell, J. A. y Chmiel, M. (2014). Generalization in and from qualitative analysis. En U. Flick (Ed.), The SAGE handbook of qualitative data analysis (pp. 540-553). Londres: Sage.

Meyer, J. (2000). Using qualitative methods in health related action research. The British Medical Journal, 320(7228), 178-181. https://doi .org/10.1136/bmj.320.7228.178

Millward, L. (2012). Focus groups. En G. M. Breakwell, J. A. Smith y D. B. Wright (Eds.). Research methods in psychology (4a ed., pp. 411-438). Los Ángeles: Sage.

Morse, J. M. (2001). Are there risks in qualitative research? Qualitative Health Research, 11(1), 3-4. https://doi.org/10.1177/104973201129118867

Neergaard, H. y Leitch, C. M. (Eds.). (2015). Handbook of qualitative research techniques and analysis in entrepreneurship. Northampton, MA: Edward Elgar.

Onwuegbuzie, A. J. y Leech, N. L. (2009). Generalization practices in qualitative research: A mixed methods case study. Quality and Quantity, 44(5), 881-892. https://doi.org/10.1007/ s11135-009-9241-z

Osborne, J. (Ed.). (2008). Best practices in quantitative methods. Thousand Oaks, CA: Sage.

Payne, G. y Williams, M. (2005). Generalization in qualitative research. Sociology, 39(2), 295-314. https://doi.org/10.1177/0038038505050540

Pfanner, E. (2006, January 2). Agencies look be- yond focus groups to spot trends. New York Times. Recuperado de http://www.nytimes .com/2006/01/02/business/media/agencieslook-beyond-focus-groups-to-spot-trends.html

Polit, D. F. y Beck, T. C. (2010). Generalization in quantitative and qualitative research: Myths and strategies. International Journal of Nursing Studies, 47(11), 1451-1458. https://doi .org/10.1016/j.ijnurstu.2010.06.004

Sanjari, M., Bahramnezhad, F., Fomani, F. K., Shoghi, M. y Cheraghi, M. A. (2014). Ethical challenges of researchers in qualitative studies: The necessity to develop a specific guideline. Journal of Medical Ethics and History of Medicine, 7, 14.

Schelmetic, T. E. (2016). Is social listening making focus groups obsolete? Recuperado de $\mathrm{http}: / /$ www.madmarketer.com/topics/agency/ articles/426047-social-listening-making-focusgroups-obsolete.htm.

Shadish, W. R., Cook, T. D. y Campbell, D. T. (2002). Generalized causal inference: A grounded theory. Boston, MA: Houghton Mifflin.

Stake, R. E. (2010). Qualitative research: Studying how things work. Nueva York: Guilford Press.

Suddaby, R. (2006). From the editors: What grounded theory is not. Academy of Management Journal, 49(4), 633-642. https://doi .org/10.5465/amj.2006. 22083020

Trauth, E. M. (2001). Qualitative research in IS: Issues and trends. Hershey, PA: Idea Group.

Urquhart, C. (2013). Grounded theory for qualitative research: A practical guide. Londres: Sage. https://doi.org/10.4135/9781526402196

Urquhart, C. y Fernández, W. (2013). Using grounded theory method in information systems. Journal of Information Technology, 28(3), 224-236. https://doi.org/10.1057/jit.2012.34

Waller, V., Farquharson, K. y Dempsey, D. (2016). Qualitative social research: Contemporary methods for the digital age. Londres: Sage.

Recibido: 17 de diciembre de 2018

Revisado: 14 de enero de 2019 Aceptado: 4 de febrero de 2019 